NASA-CR-201452

\title{
FAST INTERRUPT PLATFORM FOR EXTENDED DOS
}

\author{
T. W. Duryea \\ Rocketdyne Division, Rockwell Intemational Corp. \\ Canoga Park CA
}

\begin{abstract}
Extended DOS offers the unique combination of a simple operating system which allows direct access to the intermpt tables, 32 bit protected mode access to a $4096 \mathrm{MByte}$ address space, and the use of industry standand $C$ conpilers. The drawback is that fast Interrupt bandling requires both 32 bit and 16 bit versions of eacb real-time process internpt handler to avoid mode switches on the internupts. A set of tools has been developed which automates the process of transforming the output of a standard 32 bit $C$ compiler to 16 bit interrupt code which directly bandles the real mode internupts. The entire process compiles one set of source code via a make file, which boosts productivity by making the management of the compilo-link cycle very simple. The software components are in the form of classes written mostly in $C$. $A$ foreground process writcen as a conventional application which can use the standard $C$ libraries can communicate with the background real-time classes via a message passing mechanism. The platform thus enables the integration of high performance real-time processing into a conventional application framework.
\end{abstract}

\section{INTRODUCTION}

Various operating systems are available to facilltate the programming of real-time systems on standard IBM PC compatible hardware and other hardware. Most of thesc options suffer the drawback of 16 bit operation and relinnce on specialized development tools (i.e., compilers and linkers). The methodology presented bero originated from a neophyte's need to write a fast control systeat for a specialized imaging system which images Hydrogen leaks near a rocket engine. It has become apparent that the components already written are usable in a variety of applications. Two examples of situations where the software could be quickly adapted are an infrared scene generator which requires fast control of an optical scanner to generate scene objects and an imaging system to quickly inspect metalic surfaces for subtle defects such as scratches. The software is also embeddable, as Pbar Lap bas available an embedded version the $3861 D O S$. The trend in acrospace hardware in fact

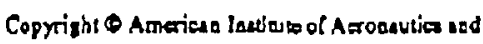
Actronsuldes, Ise. 1993. All righu reverved. seems to be towards supplying cmbedded software modules on various hardware assemblies.

Work prior to the present developments was on a 16 bit platform. After receiving advice from several sources concerning the necessity of using expensive, specialized, or unobtainable hardware and software platforms to bost real-time development work, it was decided that the standard 286 PC computer offered the advantages of availabllity, simplicity, and standard software development tools which were cheap and which worked well. A control system was written using Microsoft QuickAssembler. The control system worked well, although the software was somewhat cumbersome. The architecture was a simple interrupt driven bockground process for both a millisecond unce intemupt and a serial port internipt. The bulk of the program was a foreground process wbich was written as an ordinary application, and which commuticated with the background processes via message passing functions called in a loop in the sbell input routine which serviced the keyboard.

Several observations were made in the course of the 16 bit work. The use of standard compilers is desirable oue to commercial presstures to produce a reliable product for mass consumption. The use of standard hardware is desirable in times of reduced budgets and of the necessity of rapid response to new challenges. The use of stub interrupt handlers is useful as these handlers can call handlers written in $C$ as ordinary functions, which allows most of the difficult work to bo written in $C$. The avoidance of special $\mathrm{C}$ keywords (such as interrupt) sbould be avoided, as bugs and lack of standardization across compllers will become a problem. The uso of a MAKE file is important; lack of abillty to recompile a fresh working set of code leads to serious bug problems and is a drain on productivity. Finally, the existence of some sort of coherent framework for the programming effort boosts productivity, eliminates many serious and timeconsuming bugs, and most importantly offers modularity and reusabllity.

The above changes were implemented when the software was converted to 32 bit 386 operation. Previous familiarity with the Phar Lap 386IDOS 
extender product ${ }^{1}$ suggested that it would be a good platform for 32 bit real-time development. The product is straightforward and reliable, and has a simple and fast interrupt structure which is well documented. The Phar Lap extender does not run the real mode portion of a program in virual 86 mode, which avoids timing problems with things sucb as indirect internupt vectoring. If an application books a real mode or protected mode interruph, it gains control directy through an extender stub handler which changes the ring level if necessary. Thus one can write real-ime 32 bit code almost as easily as one would for 16 bit usage. except for the problem of mode switches.

\section{SOFTWARE ARCHITECTURE}

High performance real-time software under extended DOS requires a minimal number of mode switches during process interrupts, especially when fast (millisecond or less) Interrupt rates are Involved. The usual method for avoiding mode switches is to write separate real mode and protected mode tandlers for a softwart component. This approach generates efficient code but bas the drawback that two versions of the same algorithm must be kept congnuent. Also, it is difficult to write the real mode code in any language except assembly language. These constraints make it difficult develop real mode components in a productive manner and greatly increase the risk of bugs which are difficult to isolate.

The software arcbltecture detailed here is driven by the necessities of writing blgh performance real-ime code in $\mathrm{C}$. of compiling through a make file, of being able to make changes simply without generating bugs, of baving 32 blt access to memory, and of component reusability. The two critical ingredients of the architecture are the ability to compile a .C source filo to run in real mode and the adoption of what is essenclally an object oriented structure to the components.

Thus far the compilers used in this work are the MetaWare HighC compiler and the Phar Lap 386ASM assembler. The MetaWare compiler bas an optlon which generates assembly source files. The files will not directly assemble without problems, ws correct assembly of the files is currently not suppored. Also, the instructions in the files are nol necessarlly the sume object code instructions generated by the compiler. Nonetheless several non-trivial classes were written using these assembly source flies.
It was noticed upon early examination of the MetaWare assembly files that the instruction sequences contained within would assemble real mode code if a number of subtle changes were made to the files. The principal change is to change the segment declaration lines (containing the SEGMENT keyword) to change the segment names and conver the segments to USE16 'CODE' atuributes. All standard 32 bit 80386 instructions using any addressing mode will assemble for USE 16 segment operation. The only constraint is that any dereferenced address offset which exceeds $64 \mathrm{~K}$ will generate a processor exception In real mode.

Fig. I portrays the execution flow of a real-rime interrupt under the new architecture. One real mode stub handier and one protected mode stub bandler (written in assembler) are installed to intercept all realtime hardware internupts at several different entry points. Because the Phar Lap handler provides a fresh stack, the stub handlers are reentrant. There are no mode switches in the interrupt generation process. The invoked stub handler in tum calls the class interrupt handler implied by the interrupt vector. Each real mode and protected mode class bandler pair is derived from one .C source file. The handlers are ordinary $C$ functions which ise no special keywords at all (which avoids the problems associated with obscure keywords). The process of generating two bandlers from one source flie is termed "dualing", and the bandiers aro referred to as dual mode functions.

A hardware interrupt either occurs while the processor is in real mode or protected mode. The static data addressed by a class handier must be addressable in either mode; it is thus necessary to bave all static data used by the handlers resident in conventional memory (i.e., below I MB). Both of the dual mode handlers should execute precisely the same algorithm on precisely the same dota. Fig. 1 shows that the class static data is arranged as instance slots grouped into class slots which are bundled with the real-mode code. Fig. 2 provides more detail on the conventional memory arrangement. The simplest way to get this arrangement loaded into conventional memory is to use a simple assembly source file to force the segment ordering. (which gets the whole fig. 2 arrangement Into the bottom of the load image) and to use the REALBREAK extender switch to ensure that the necessary portion gets loaded below $1 \mathrm{MB}$.

Fig. 3 displays the pars of a real-time class. Typically a trivial assembly source file declares space for $\mathrm{N}$ instances to a segment named REALDATA of class

2

American Institute of Aeronautics and Astronautics 
'CODE'. A dual mode.C source file contains the handler source code and code for specialized dual mode functions. These functions may be called by the handler and by other real-time software classes. Dual mode functions which operate on static data must bave some kind of semaphored access to the daka, as the functions must not operate on stacle data when a bandler already operating on the data is itself internupted. The class handlers are written without regards to possible interruption (except where timing problems are a concern), but some dual mode functions can possibly return with a Data_Access_Denied return code. The calling process must be able to work around this.

The protected mode class functions (1.e., the foreground functions) in fig. 3 are conventional $C$ functions which present the bigh level class interface to the conventional part of the application, called the foreground process. The real-tiene classes form the part of the application called the background processes. The division of the application into these two parts makes the majority of the work no more difficult than writing an ordinary $C$ program. Thus the kind of reusability associated with object arlented programming is imparted to the real-ime components. Development of the real-time classes is simplified due to the fact that one is wriling ordinary $\mathrm{C}$ code.

Most non-trivial applications require some sort of communication between the foreground and background on a regular basis. Two examples are the need to drain and process serial port input and the need to transfer interrupt trace debugging data from a small conventional memory buffer to a much larger extended memory trace buffer. A simple way to ensure this communication is to execute a servicing function in a keyboard input loop, which allows a shell-driven user input mode. Any long foreground execution path should call the same function at various times. Another possibility is to book the timer tick to always gain control in protected mode and call the servicing fuaction.

\section{IMPLEMENTATION DETAILS}

The following details relate to the software architecture implementation. One important consideration is that the procedure used to generate real mode code from the .C source file is general in the sense that only one algorithm is needed to convert alt files: i.e., only one single-pass editor need be written. The details regard only the use of the MetaW are compiler.
The real-time stub handlers are part of a special singleinstance class named is (which mimics a $\mathrm{CH}$ PUBLIC base class). The role of IS is to provide a standard interface for installation and usage of the rel-time classes. When a real-dime class instance constructs, it provides its inberited IS base class tho interrupt vector, the addresses of both dual bandlers, and a pointer to its conveotional memory static data. The IS class stores both real mode and protected mode far addresses of these quantities in tables for quick access by the stub handlers. The only class bandler access to static data is througb its structure pointer.

The current constaint on conventional memory length is that all of the code be less than $64 \mathrm{~K}$ in length. If far data pointers in the $C$ source are used, the IS stack pool may be up to $64 \mathrm{~K}$ in length and any class instance may be up to $64 \mathrm{~K}$ in length. If near data pointers are used, the stack pool and all class instance conventional static data must fit in less than $64 \mathrm{~K}$. Regarding for pointer usage, MetaWare and Watcom compliers support the far keyword, while Microsoft and Borland do not. Also, use of far pointers in C code may result in many segment loading instructions, which slow down execution considerably. (Class bandlers rewritten in assembly language need only set the ES register once per pass and use segment overrides.) In general, a good strategy is to make the conventional memory usage small and use near pointers.

The role of the stub handlers is mainly to secure a stack from a stack pool, to call the approprtate class handler, and to send the EOI signal and return. The stack pool is not necessary (the Phar Lap handler uses a fresh stack), but facilitates debugging, as the stacks exist in the application dala segment. The stub bandler pusbes a far pointer to the correct static data instance and calls the bander function. Several quantities such as the interrupted address are pushed before the static data pointer, which allows ready access of debugging parameters to the class bandlers.

If the called class bandler uses near pointers, the data addressing is successful, as the real mode DS reglster and SS register boch point at or just below the beginning of the stack pool portion. The real mode static data pointer offset is fixed up to this value. If far pointers are used, the real mode DS points just below the correct instance, allowing 64K per instance. It is important when calling the real mode class handler that two padding bytes be pusbed just before the call instruction, to maintain the proper stack frame across the call. 
To generate the dualed real mode 32 bit object code the following procedure works: Irst the $\mathrm{C}$ compiler is run to generate the assembly output file, which should bave a different root file name. The file is then processed by a single pass editor which regenerates an aitered flle which then is assembled by the assembler. The make rile ensures that any changes to the .C source file generates new copies of both object code files.

The singlo pass editor was written to read in a text file to 2 beap. A directory contains the offsets of each text line, which makes the editing process straightforward.

The following set of commands has been found to convert MetaWare HighC.S assembly output files successfully. The following lines should be included into the dual mode files:

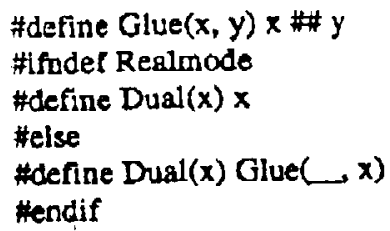

Taus a function int func(int arg) declared as int Dual(func)(int arg) will obtain the rame _func when the switch-DRealmode is used on the compllation. This prevents name recurrences. The function should be called in the form Dual(func)(value):.

The exact set of peeded editor commands changes from release to release:

1) Change the "TITLE nane.c" line to "TITLE mame. $c^{*}$ to not confuse the librarlan.

2: Replace the "_TEXT segment" line with "REALTEXT SEGMENT PUELIC DWORD USE16 'CODE'" to change the segment declaration.

3: Change the "_TEXT ends" line to " REALTEXT ENDS".

4: Delete the "CONST segment" and "CONST ends" lines (there should be no literal strings in the source flle).

5: Insert the line ".386" near the file beginning.

6: Delele the lines "extm _mwargstack", "extm _mwargstack:NEAR", "extm_mwgoc", and "extrn_mwgoc:NEAR" to prevent warnings.

7: Replace the line "CGROUP GROUP_TEXT" with "CGROUP GROUP REALTEXT"
8: Replace the line "DGROUP GROUP" with "DGROUP GROUP _DATA".

9: Move lines containing the word "extm" such that they follow the next line which contains the word "public".

10: Fot all lines containing the word "call", precede the line by "sub esp, 2 ", and follow it by "add esp. 2 ". This aligns the stack frame for calls to dual mode functions.

11: Replace the lines "leave" with the set of lines "mov esp, ebp" and "pop ebp". This prevents the 16 bit form of leave from being assembled.

12: Delete the lines "mov eax,ds".

13: Replace the lines "mov es, eax" with the pair of lines "mov ax, ds", "mov es, $\mathrm{ax}^{\mathrm{r}}$ to facilitate tar pointer usage.

14: Replace the lines "les ecx,dword ptr $8[$ ebp]" with "Ies ecx, FWORD PTR [ebp][8]" to facilitate far pointer usage.

The above dualing procedure was used to write several real-time classes, most notably a $8250-16550$ serial por interface which could bo called from within other real-time classes. The required set of editor commands required little maintenance as the amoune of bigh level source code increased, which was the boped-for result when the work began. Some additions need to be made to facilitate things such as argument passing when dualed functions are called. The only assembler instructions in MetaWare which never worked involved bit thelds. The changes needed above do not correct errors in the outputted assembler instructions, but in fact ensure that 32 bit instructions are not misinterpreted within a USE16 segment. Obviously an assembly output file which is guaranteed to assemble correct object code (identlcal to .OBJ output) and output instructions which speciflcally refer to 32 bte operations would be ideal.

A number of simple considerations must be kept in mind as dual mode.C files are being written. Stack checking must be off. A bandler should have a static data structure pointer as its sole argument. unless debugging arguments are to be added. No standard library functions can be called from dual mode functions (all called functions must be of dual mode form). Tbings such as function pointers must be bandled carefully. The volatile keyword should be used on the static dnta structure, but this alone will not prevent instruction overhang which causes seal-time bugs. For example, suppose a one-bit flag is to be cbanged in a tlags word. High level compilers typically copy the word to a register, alter the register, thea replace the word. If a flag changing interrupt 
occurs during this sequence, the word changed by the internpt will be overwritten by the interrupted process. The volatile keyword will reduce the instruction overtang, but will not eliminate 1t. These bugs are prevented by knowing the bebavior of the compller and using short (assembly) functions with names such as cl_iO and st_iO to control the interrupt flag when necessary. Good design technique can avoid many situations where overhang could be a problem.

The fastest way to develop a new class is to start with a trivial set of working code and test a minimal set of interacting closses $\mathrm{x}$ incremental changes are made to the new class. Ironically, a slow machine such as a 386 belps out, as the processor is more eavily overwhelmed than a faster processor. Rellability of the class is enhanced by driving the test progran at a rate which generates overhead problems. The class may be made fault tolerant to many of the errors which occur. Such behavior enbances the reliability of the class when it interacts with other classes later on. The ability to store large amounts of trace debugging information and to have access to interrupt stacks, interrupted addresses, and timing information can help pinpoint subtle class interaction bugs. All internupt trace debugging features stould be triggered on a single *define to allow the complete removal of the feature.

The implementation of this compilation technique using other compilers should be straightforward, although the task has yet to be perforned. The assembly output files of the compiler must be examined, and a new set of editor commands which converge to one set of universal commands must be identified. Most standard 32 bit compilers don't support the far keyword or the int386 function calls. Most 32 bit compilers and debuggers which nun on the Phar Lap extender are Windows NT products or
WIN32S products, which cause problems with the obsolescent REALBREAK switch. A lack of reliance on this switch is therefore destrable.

\section{CONCLUSIONS}

The methodology described here has been implemented and is known to work. The tasks of converting to other major $C$ compilers and of avoiding REALBREAK have pot been implemented, but aro expected to be straightforward. The actual speed or maximum Intorrupt rate of an application depend on the nature and complexity of the processes involved. This arcbitecture has the property that the overhead is mintmized in a system wbich provides a uniform framework for installing real-time components. Thus as the $\times 86$ processor family advances programs written in this platform gain the some speed increases.

Several classes bave been written besides the IS intertace class. They include a real-timo serial port class and a trace buffer debugging class which can store large amounts of interrupt trace data. A simple scheduler class to administrate several control system algorithms is under dovelopment. The classes are easily reused in other applications.

\section{ACKNOWLEDGMENT}

The work reported here was supported by NASA Marsball Space Figbt Center (contract NAS8-40000).

\section{REFERENCE}

Baker, M. Steven, and Schulman, Andrew, "80386based Procected Mode DOS Extenders", in Extending DOS.Rry Duncan, ed.), Addison-Wesley Publishing Company, Inc., Reading, Mass, p. 193, 1990. 
FIG. 1: HARDWARE INTERRUPT ARCHITECTURE

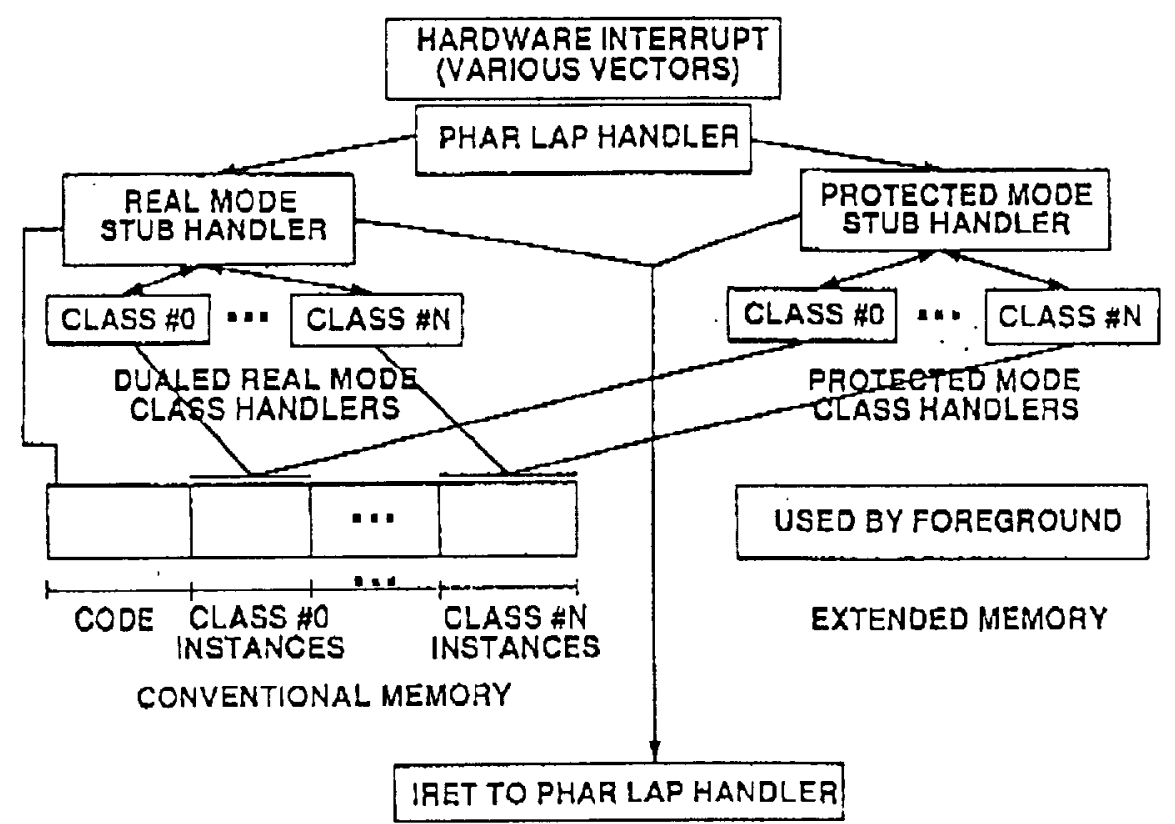

\section{FIG. 2: CONVENTIONAL MEMORY MAP}

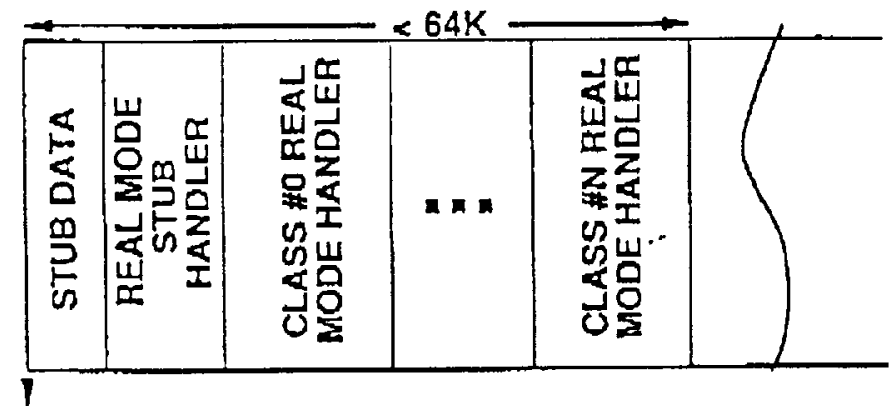

CS: 0000 (PROTECTED)

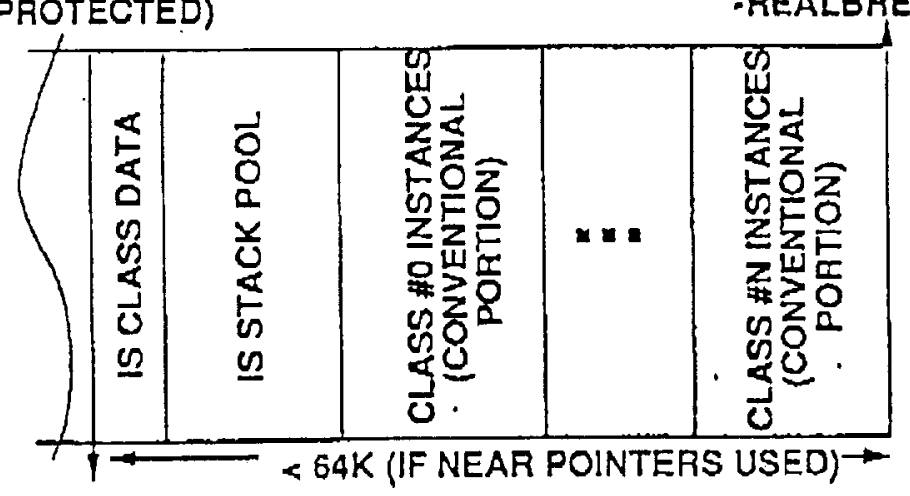

DS:0000 (REAL MODE)

6

American Institute of Aeronautics and Astronautics 


\section{FIG. 3: REAL-TIME CLASS STRUCTURE}

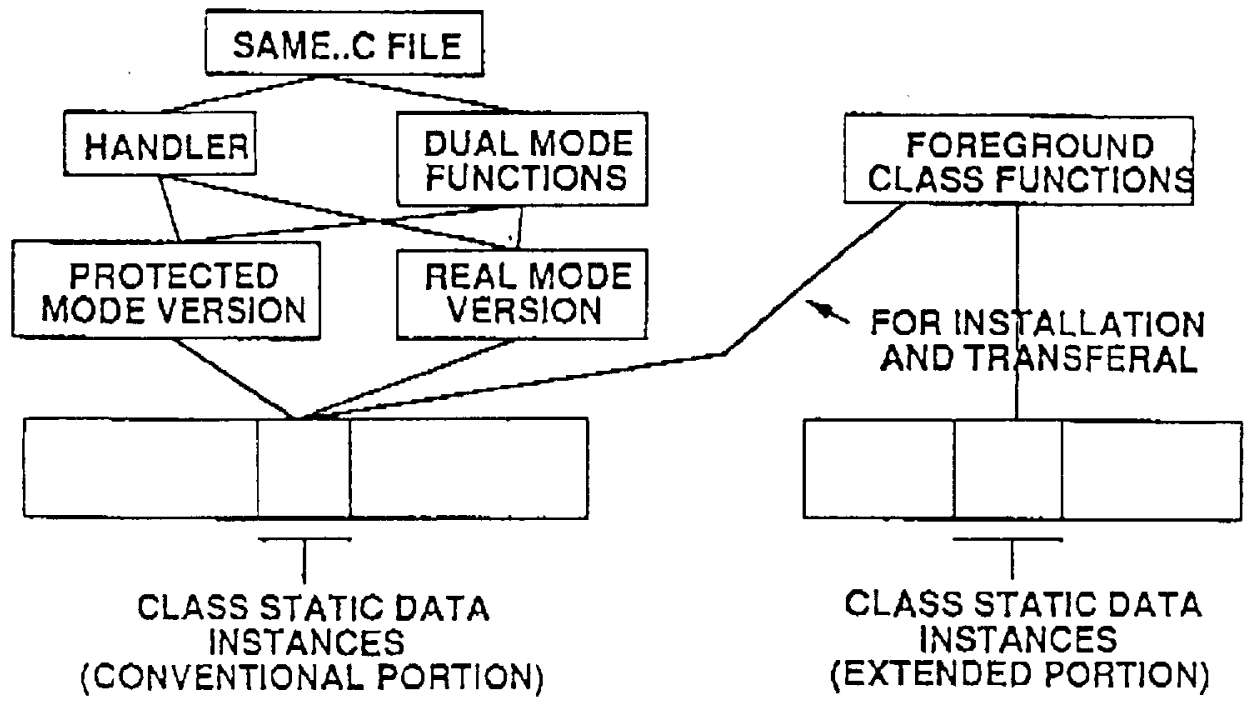

